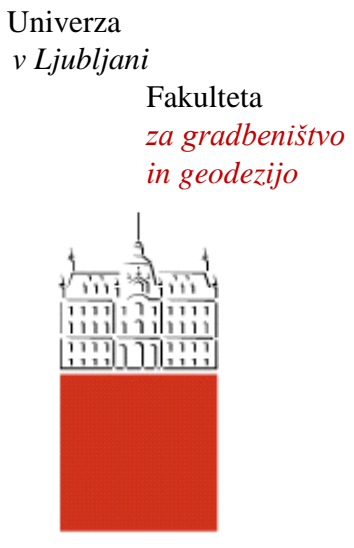

Jamova cesta 2

1000 Ljubljana, Slovenija

http://www3.fgg.uni-lj.si/

\section{DRUGG - Digitalni repozitorij UL FGG http://drugg.fgg.uni-lj.si/}

Ta članek je avtorjeva zadnja recenzirana različica, kot je bila sprejeta po opravljeni recenziji.

Prosimo, da se pri navajanju sklicujte na bibliografske podatke, kot je navedeno:
University
of Ljubljana

Faculty of Civil and Geodetic Engineering

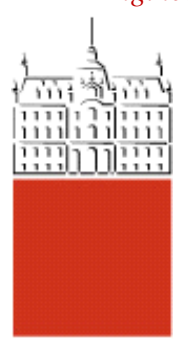

Jamova cesta 2 SI - 1000 Ljubljana, Slovenia http://www3.fgg.uni-lj.si/en/

DRUGG - The Digital Repository http://drugg.fgg.uni-lj.si/

This version of the article is author's manuscript as accepted for publishing after the review process.

When citing, please refer to the publisher's bibliographic information as follows:

Zupan, D., Saje, M. 2003. The three-dimensional beam theory: finite element formulation based on curvature. Computers \& Structures 81: 1875-1888. DOI: 10.1016/S00457949(03)00208-6. 


\title{
The Three-Dimensional Beam Theory: Finite Element Formulation based on Curvature
}

\author{
D. Zupan, M. Saje* \\ University of Ljubljana, Faculty of Civil and Geodetic Engineering, Jamova 2, \\ SI-1115 Ljubljana, Slovenia
}

\begin{abstract}
The article introduces a new finite element formulation of the three-dimensional 'geometrically exact finite-strain beam theory'. The formulation employs the generalized virtual work principle with the pseudo-curvature vector as the only unknown function. The solution of the governing equations is obtained by using a combined Galerkin-collocation algorithm. The collocation ensures that the equilibrium and the constitutive internal force and moment vectors are equal at a set of chosen discrete points. In Newton's iteration special update procedures for the pseudocurvature and rotational vectors have to be employed because of the non-linearity of the configuration space. The accuracy and the efficiency of the derived numerical algorithm are demonstrated by several examples.
\end{abstract}

Key words: three-dimensional beams, three-dimensional rotations, curvature, finite element method.

$P A C S$ :

\section{Introduction}

Engineering structures are often modelled by beam models. In the present paper, we limit ourselves to models, derived from the resultant forms of the differential equilibrium equations. Their strain-displacement equations consist of three displacement components and six strain measures (longitudinal and shear strains, and pseudo-curvatures) of the axis of the beam. The strain measures are derived in such a way that the relationships between the displacements, the strains, and the stress resultants are consistent with the virtual

* Corresponding author. Tel.: +386 14768 613; FAX: +38614768629

Email address: msaje@fgg.uni-lj.si (M. Saje). 
work principle at the deformed state for any magnitude of displacements, rotations and strains. The model is often called the 'geometrically exact finitestrain beam theory' (Simo [23], Simo and Vu-Quoc [24]) although both its exactness ( $\mathrm{Li}[15])$ and its applicability to finite strain regime may be questioned.

Because the spatial rotations - which play a crucial role in the beam models are elements of a multiplicative group, the configuration space of deformations is a non-linear manifold. The way the rotations are parametrized is essential, as a particular selection of the parametrization has a direct influence on the algorithm and the form of the tangent stiffness matrix.

In contrast to previous formulations, which base the finite element implementation of the geometrically exact beam theory on both displacements and rotations as the interpolated degrees of freedom (as, e.g., Simo and Vu-Quoc [24], Ibrahimbegovic [9], Crisfield [4]), or solely on rotations (Jelenić and Saje [13]), the present finite element implementation of geometrically exact 3D beam theory parallels the ones given in [18] and [19] for plane frames. The pseudo-curvature vector is used as the only degree of freedom that needs to be interpolated along the element, while the displacement and rotational components are not interpolated. This 'one-field' formulation not only results in the fact that the locking never occurs (that is also the characteristic of finite elements by Jelenić and Saje [13]), but also provides enhanced accuracy for the same number of degrees of freedom compared to displacement-based finite elements, and further enables more realistic description of stress distributions within the beam element; this is of the utmost importance in describing the behaviour of non-linear material in the state of localized strain and stress (e.g., in a plastic hinge).

In standard finite element formulations, as is well known, the stress-resultants, obtained from the equilibrium equations, and those calculated from the constitutive equations, are not equal. Here, this 'inconsistency of equilibrium at cross-sections' is solved by enforcing the consistency condition to be satisfied in a set of predefined points (here taken to coincide with the interpolation nodes) (the 'collocation'). A similar strategy was employed by Vratanar and Saje [31] for elastic-plastic analysis of plane frames. In the present formulation, the determination of internal forces does not require the differentiation with respect to the arc-length, $x$. This is a notable advantage compared to formulations, where the derivatives with respect to $x$ are needed for the evaluation of internal forces, since the differentiated quantities are as a rule one order less accurate than the quantity itself. 


\section{Geometry and kinematics of the three-dimensional beam}

Geometry of the three-dimensional beam is described by the line of centroids of cross-sections and by the family of the cross-sections not necessarily perpendicular to the line of centroids. The geometric shape of the cross-sections is assumed to be arbitrary and constant along the beam. A cross-section is assumed to suffer only rigid rotation during deformation. The line of centroids is described by the position vector $\vec{r}$ (see Figure 1). In order to describe positions of the cross-sections, a family of orthonormal vector bases $\left\{\vec{G}_{1}, \vec{G}_{2}, \vec{G}_{3}\right\}$, here called the 'material basis', is introduced. Vectors $\vec{G}_{2}$ and $\vec{G}_{3}$ are directed along the principal axes of inertia of the cross-section, and $\vec{G}_{1}$ is normal to the cross-section: $\vec{G}_{1}=\vec{G}_{2} \times \vec{G}_{3}$. In general, the basis $\left\{\vec{G}_{1}, \vec{G}_{2}, \vec{G}_{3}\right\}$ is different at each point of the line of centroids. It is found convenient to distinguish between the reference (undeformed) configuration and an arbitrary deformed configuration. That way we can express the vectors $\vec{r}, \vec{G}_{1}, \vec{G}_{2}$, and $\vec{G}_{3}$ as functions of parameter $x$, the arc-length of the reference line of centroids of cross-sections. Note that the reference configuration, described by $\vec{r}^{0}$ and $\left\{\vec{G}_{1}, \vec{G}_{2}^{0}, \vec{G}_{3}^{0}\right\}$, is only a special case of an arbitrary deformed configuration.

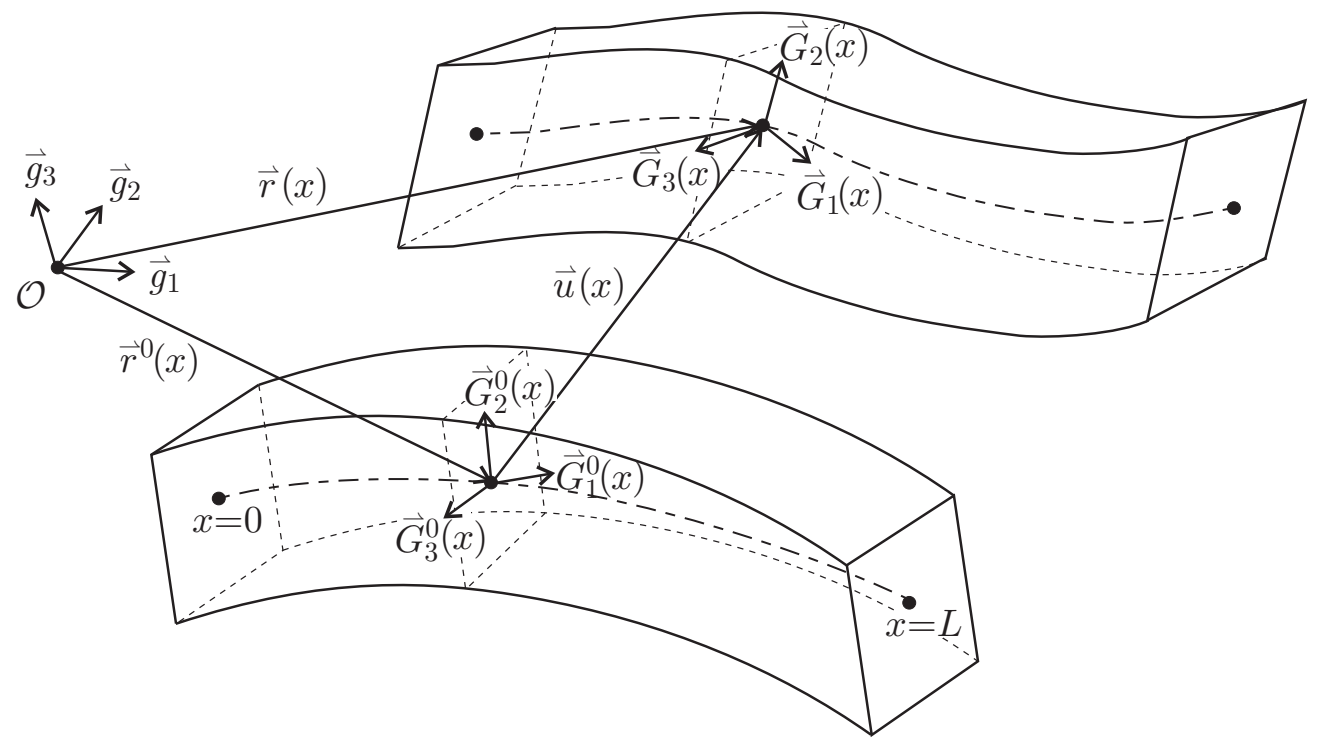

Fig. 1. Kinematics of the reference and the deformed configurations of the beam.

In addition to the material basis, the 'spatial basis' with orthonormal base vectors $\left\{\vec{g}_{1}, \vec{g}_{2}, \vec{g}_{3}\right\}$ is introduced, which spans the physical space in which the beam is embedded (the three-dimensional Euclidean space in mathematics). The material basis is related to the spatial basis by the rotation matrix 
$\mathbf{R}(x)$, which maps $\left\{\vec{g}_{1}, \vec{g}_{2}, \vec{g}_{3}\right\}$ into $\left\{\vec{G}_{1}(x), \vec{G}_{2}(x), \vec{G}_{3}(x)\right\}$. The deformed geometry and the position of the beam in space are fully described by $\vec{r}(x)$ and $\mathbf{R}(x)$. The rotation matrix $\mathbf{R}(x)$ has nine components, of which only three are independent. It is convenient to parametrize the components of $\mathbf{R}$ by the rotational vector $\vec{\vartheta}[1]$. The rotational vector is a vector that lies on the axis of rotation with its length equal to the angle of rotation.

In what follows, the abstract vectors will be expressed by three scalar components with respect to the material or spatial orthonormal basis. A vector, $\vec{v}$, can be expressed in a vector form

$$
\vec{v}=v_{g 1} \vec{g}_{1}+v_{g 2} \vec{g}_{2}+v_{g 3} \vec{g}_{3}=v_{G 1} \vec{G}_{1}+v_{G 2} \vec{G}_{2}+v_{G 3} \vec{G}_{3}
$$

or in a matrix form

$$
\boldsymbol{v}_{g}=\left[\begin{array}{c}
v_{g 1} \\
v_{g 2} \\
v_{g 3}
\end{array}\right], \quad \boldsymbol{v}_{G}=\left[\begin{array}{c}
v_{G 1} \\
v_{G 2} \\
v_{G 3}
\end{array}\right],
$$

as a one-column matrix. An arbitrary abstract vector $\vec{v}$ can be expressed with respect to material or spatial basis, but when given in the matrix form only the operations between the vectors, expressed with respect to the same bases, are allowed. Therefore, the relationship between one-column matrices $\boldsymbol{v}_{g}$ and $\boldsymbol{v}_{G}$ is of great importance. The base vectors $\vec{G}_{i}$ are expressed as the image of the rotation matrix on the spatial basis:

$$
\vec{G}_{i}=\mathrm{R}_{1 i} \vec{g}_{1}+\mathrm{R}_{2 i} \vec{g}_{2}+\mathrm{R}_{3 i} \vec{g}_{3}, \quad i=1,2,3,
$$

where $\mathrm{R}_{i j}$ denotes a component of rotation matrix. Inserting (2) into (1) gives the relationship between the two one-column matrix presentations of a vector

$$
\boldsymbol{v}_{g}=\mathbf{R} \boldsymbol{v}_{G}
$$

which is commonly referred to as the coordinate transformation equation. In what follows, one-column matrices $\boldsymbol{v}_{g}$ and $\boldsymbol{v}_{G}$ will, for simplicity of language, be termed vectors.

Remark 1 Another meaning of the rotation matrix is revealed in (3). It rotates spatial basis into material, but it also transforms a one-column matrix of a vector, expressed with respect to the material basis, into its spatial basis presentation.

The relationship between the rotation matrix $\mathbf{R}$ and the rotational vector $\boldsymbol{\vartheta}$ 
is described by the Rodrigues formula

$$
\mathbf{R}=\mathbf{I}+\frac{\sin \vartheta}{\vartheta} \boldsymbol{\Theta}+\frac{1-\cos \vartheta}{\vartheta^{2}} \boldsymbol{\Theta}^{2},
$$

where $\mathbf{I}$ means the unit matrix, $\vartheta=\|\boldsymbol{\vartheta}\|$ is the Euclidean norm of the rotational vector $\boldsymbol{\vartheta}$, and $\boldsymbol{\Theta}$ is the skew-symmetric matrix composed from the components of $\vartheta$

$$
\boldsymbol{\vartheta}=\left[\begin{array}{l}
\vartheta_{1} \\
\vartheta_{2} \\
\vartheta_{3}
\end{array}\right] \rightarrow \boldsymbol{\Theta}=\left[\begin{array}{ccc}
0 & -\vartheta_{3} & \vartheta_{2} \\
\vartheta_{3} & 0 & -\vartheta_{1} \\
-\vartheta_{2} & \vartheta_{1} & 0
\end{array}\right]
$$

Remark 2 The parametrization of rotations with the rotational vector is introduced in (4) in order to reduce 9 constrained parameters into 3 arbitrary ones. There is a number of possibilities for choosing three independent rotational parameters (see, e.g., [1], [2], [8]). In principle, a unique global representation of finite rotations needs at least five parameters. Nevertheless, the three-parameter rotational vector chosen here has proved to be adequate for the description of large rotations in our finite element formulation when correctly implemented in the computer code.

\section{Strain measures, stress resultants and constitutive equations}

In this section we introduce the strain vectors $\boldsymbol{\gamma}$ and $\boldsymbol{\kappa}$, following the approach of Reissner [20] and Simo [23]. The strain vectors are related to the previously described kinematic quantities: the position vector $\boldsymbol{r}$ and the rotational vector $\boldsymbol{\vartheta}$. Vectors $\boldsymbol{\gamma}$ and $\boldsymbol{\kappa}$ are given here with respect to the material basis, while the vectors $\boldsymbol{r}$ and $\boldsymbol{\vartheta}$ are expressed with respect to the spatial basis. Using the principle of virtual work, together with equilibrium equations of the beam, yields the relationships between the variations of the vectors of kinematic quantities $(\boldsymbol{r}, \boldsymbol{\vartheta})$ and strains $(\boldsymbol{\gamma}, \boldsymbol{\kappa})$

$$
\begin{aligned}
\delta \boldsymbol{\gamma} & =\mathbf{R}^{T}\left(\delta \boldsymbol{r}^{\prime}-\delta \boldsymbol{\vartheta} \times \boldsymbol{r}^{\prime}\right) \\
\delta \boldsymbol{\kappa} & =\mathbf{R}^{T} \delta \boldsymbol{\vartheta}^{\prime}
\end{aligned}
$$

The prime (') denotes the derivative with respect to arc-length parameter $x$, and " $X$ " is a vector product.

Remark 3 Note that the variation of a one-column matrix (vector), given with respect to the material basis, is a relative variation of a vector that assumes only the variation of components. This is in accord with the notion of 'objective rates'; see e.g. [23]. 
The integration of equations (5) and (6) gives strain measures $\boldsymbol{\gamma}$ and $\boldsymbol{\kappa}$ as functions of displacements and rotations

$$
\begin{aligned}
\boldsymbol{\gamma} & =\mathbf{R}^{T} \boldsymbol{r}^{\prime}+\boldsymbol{c} \\
\boldsymbol{\kappa} & =\mathbf{R}^{T} \boldsymbol{\omega}+\boldsymbol{e} .
\end{aligned}
$$

Vector functions $\boldsymbol{c}(x)$ and $\boldsymbol{e}(x)$ are variational constants $(\delta \boldsymbol{c}=\mathbf{0}, \delta \boldsymbol{e}=\mathbf{0})$ to be determined from the known strains and kinematics in the beam at the reference configuration. One-column matrix $\boldsymbol{\omega}$, introduced in (8), is the axial vector of the antisymmetric matrix $\boldsymbol{\Omega}=\mathbf{R}^{\prime} \mathbf{R}^{T}$. Its components are given with respect to the spatial basis. In rigid-body dynamics, where time $t$ plays the role of parameter $x, \boldsymbol{\omega}$ is commonly referred to as the angular velocity vector. In the differential geometry of curves, $\boldsymbol{\omega}$ is referred to as the curvature. Due to obvious similarity with the latter, $\boldsymbol{\omega}$ could here be termed the curvature. Yet $\boldsymbol{\omega}$ is not the curvature of the centroidal axis of the beam, so the term 'pseudo-curvature' is more adequate. For further descriptions of the angular velocity vector see, e.g., [1], [2], and [5].

For the reasons which will become clear later, an additional strain measure, $\boldsymbol{\kappa}^{*}$, is introduced. Let us define the vector $\delta \boldsymbol{\kappa}^{*}$ by the equation

$$
\delta \boldsymbol{\kappa}^{*}=\mathbf{R} \delta \boldsymbol{\kappa} .
$$

Inserting equation (9) into equation (6) and integrating in the sense of variations, yields

$$
\kappa^{*}=\boldsymbol{\vartheta}^{\prime}+\boldsymbol{d} .
$$

Here vector function $\boldsymbol{d}(x)$ marks a variational constant $(\delta \boldsymbol{d}=\mathbf{0})$, obtained from the shape of the beam in the reference configuration.

The relationship between $\boldsymbol{\kappa}^{*}$ and $\boldsymbol{\kappa}$ is also needed for future use. It is obtained by employing the known relationship between $\boldsymbol{\omega}$ and $\boldsymbol{\vartheta}^{\prime}$. The development of the relationship is rather lengthy and can be found, e.g., in Atluri and Cazzani [2]. The result can be written in the following form

$$
\boldsymbol{\omega}=\mathbf{T}(\boldsymbol{\vartheta}) \boldsymbol{\vartheta}^{\prime}
$$

where $\mathbf{T}(\boldsymbol{\vartheta})$ denotes a matrix given by the following expression:

$$
\mathbf{T}(\boldsymbol{\vartheta})=\mathbf{I}+\frac{1-\cos \vartheta}{\vartheta^{2}} \boldsymbol{\Theta}+\frac{\vartheta-\sin \vartheta}{\vartheta^{3}} \mathbf{\Theta}^{2}
$$

Employing (11) in (8) gives

$$
\begin{aligned}
\boldsymbol{\kappa} & =\mathbf{R}^{T} \mathbf{T} \boldsymbol{\kappa}^{*}-\mathbf{R}^{T} \mathbf{T} \boldsymbol{d}+\boldsymbol{e} \\
& =\mathbf{R}^{T} \mathbf{T} \boldsymbol{\kappa}^{*}+\boldsymbol{f} .
\end{aligned}
$$


For further use the stress-resultant vectors over the cross-section must be introduced. The stress-resultant force is denoted by $\boldsymbol{N}$ and the resulting moment vector by $\boldsymbol{M}$. Both $\boldsymbol{N}$ and $\boldsymbol{M}$ are expressed with respect to the material basis. The stress-resultants $\boldsymbol{N}$ and $\boldsymbol{M}$ are dependent on the strains $\boldsymbol{\gamma}$ and $\boldsymbol{\kappa}$ through the constitutive equations which are taken here as being given by the equations

$$
\begin{aligned}
\boldsymbol{N} & =\mathcal{C}_{N}\left(\boldsymbol{\gamma}-\boldsymbol{\gamma}_{0}, \boldsymbol{\kappa}-\boldsymbol{\kappa}_{0}\right) \\
\boldsymbol{M} & =\mathcal{C}_{M}\left(\boldsymbol{\gamma}-\boldsymbol{\gamma}_{0}, \boldsymbol{\kappa}-\boldsymbol{\kappa}_{0}\right)
\end{aligned}
$$

The non-linear operators $\mathcal{C}_{N}$ and $\mathcal{C}_{M}$ describing the material of the beam must be invariant under superposed rigid-body motions and sufficiently smooth. We assume the existence of at least the first derivatives with respect to $\boldsymbol{\gamma}, \boldsymbol{\kappa}$, and $x$.

\section{Generalized virtual work principle}

Let us assume that the beam is subjected to the external distributed force and moment $\boldsymbol{n}$ and $\boldsymbol{m}$ per unit length of the reference line of centroids; $\boldsymbol{n}$ and $\boldsymbol{m}$ are expressed with respect to the spatial basis. For the beam of initial length, $L$, the principle of virtual work may be stated in the following form:

$$
\begin{aligned}
\int_{0}^{L}\left(\boldsymbol{N}^{T} \delta \boldsymbol{\gamma}+\right. & \left.\boldsymbol{M}^{T} \delta \boldsymbol{\kappa}\right) d x=\int_{0}^{L}\left(\boldsymbol{n}^{T} \delta \boldsymbol{r}+\boldsymbol{m}^{T} \delta \boldsymbol{\vartheta}\right) d x \\
& +\left(\boldsymbol{S}^{0}\right)^{T} \delta \boldsymbol{r}^{0}+\left(\boldsymbol{P}^{0}\right)^{T} \delta \boldsymbol{\vartheta}^{0}+\left(\boldsymbol{S}^{L}\right)^{T} \delta \boldsymbol{r}^{L}+\left(\boldsymbol{P}^{L}\right)^{T} \delta \boldsymbol{\vartheta}^{L}
\end{aligned}
$$

$\boldsymbol{S}^{0}, \boldsymbol{P}^{0}, \boldsymbol{S}^{L}, \boldsymbol{P}^{L}$ are vectors of the external point loads and moments at the boundaries $x=0$ and $x=L$. The upper indices 0 and $L$ mark the value of a quantity at the fixed values of the arc-length parameter $x=0$ or $x=L$. Hence, $\delta \boldsymbol{r}^{0}$ and $\delta \boldsymbol{r}^{L}$ are variations of the position vector $\boldsymbol{r}$ at $x=0$ and $x=L$, and $\delta \boldsymbol{\vartheta}^{0}$ and $\delta \boldsymbol{\vartheta}^{L}$ are variations of the rotational vector at $x=0$ and $x=L$. It should be noted that the quantities $\boldsymbol{\gamma}, \boldsymbol{\kappa}, \boldsymbol{r}$, and $\boldsymbol{\vartheta}$ are not mutually independent, because they are constrained by the kinematic conditions (7), (8), (10), and (11). Once we eliminate $\boldsymbol{\kappa}$ and $\boldsymbol{\omega}$ by using (8) and (11), two independent equations (7) and (10) remain the constraining equations for $\gamma$, $\boldsymbol{\kappa}^{*}, \boldsymbol{r}$, and $\boldsymbol{\vartheta}$, as well as their variations. According to the method of Lagrangian multipliers in constrained problems of calculus of variations and the related work of Planinc et al. [18] on planar beams, the constraining equations

$$
\begin{array}{r}
\mathbf{R} \gamma-r^{\prime}-\mathbf{R} c=0 \\
\boldsymbol{\kappa}^{*}-\vartheta^{\prime}-\boldsymbol{d}=\mathbf{0}
\end{array}
$$

are scalarly multiplied by arbitrary, independent, at least once differentiable vector functions $\boldsymbol{a}(x)$ and $\boldsymbol{b}(x)$, given with respect to the spatial basis. The 
scalar products of the multipliers and the constraining equations are integrated along the length of the beam and varied with respect to now independent variables $\boldsymbol{a}, \boldsymbol{b}, \boldsymbol{\gamma}, \boldsymbol{\kappa}^{*}, \boldsymbol{r}$, and $\boldsymbol{\vartheta}$

$$
\begin{aligned}
& \int_{0}^{L} \delta \boldsymbol{a}^{T}\left(\mathbf{R} \boldsymbol{\gamma}-\boldsymbol{r}^{\prime}-\mathbf{R} \boldsymbol{c}\right) d x+\int_{0}^{L} \boldsymbol{a}^{T}\left(\delta \mathbf{R} \boldsymbol{\gamma}+\mathbf{R} \delta \boldsymbol{\gamma}-\delta \boldsymbol{r}^{\prime}-\delta \mathbf{R} \boldsymbol{c}\right) d x=0 \\
& \int_{0}^{L} \delta \boldsymbol{b}^{T}\left(\boldsymbol{\kappa}^{*}-\boldsymbol{\vartheta}^{\prime}-\boldsymbol{d}\right) d x+\int_{0}^{L} \boldsymbol{b}^{T}\left(\delta \boldsymbol{\kappa}^{*}-\delta \boldsymbol{\vartheta}^{\prime}\right) d x=0 .
\end{aligned}
$$

The difference of the terms $\delta \mathbf{R} \gamma$ and $\delta \mathbf{R} \boldsymbol{c}$ is transformed into a more useful form with a well known formula for the variation of the rotation matrix (see, e.g., [2]) $\delta \mathbf{R}=\delta \boldsymbol{\Theta R}$ :

$$
\delta \mathbf{R}(\boldsymbol{\gamma}-\boldsymbol{c})=\delta \boldsymbol{\Theta} \mathbf{R}(\boldsymbol{\gamma}-\boldsymbol{c})=\delta \boldsymbol{\vartheta} \times \mathbf{R}(\boldsymbol{\gamma}-\boldsymbol{c}) .
$$

The terms $\boldsymbol{a}^{T} \delta \boldsymbol{r}^{\prime}$ and $\boldsymbol{b}^{T} \delta \boldsymbol{\vartheta}^{\prime}$ are partially integrated and the relationship $\delta \boldsymbol{\kappa}^{*}=$ $\mathbf{R} \delta \boldsymbol{\kappa}$ is employed. We obtain

$$
\begin{array}{r}
\int_{0}^{L} \delta \boldsymbol{a}^{T}\left(\mathbf{R} \boldsymbol{\gamma}-\boldsymbol{r}^{\prime}-\mathbf{R} \boldsymbol{c}\right) d x+\int_{0}^{L} \boldsymbol{a}^{T} \mathbf{R} \delta \boldsymbol{\gamma} d x+\int_{0}^{L} \boldsymbol{a}^{T}(\delta \boldsymbol{\vartheta} \times \mathbf{R}(\boldsymbol{\gamma}-\boldsymbol{c})) d x \\
-\left[\boldsymbol{a}^{T} \delta \boldsymbol{r}\right]_{0}^{L}+\int_{0}^{L}\left(\boldsymbol{a}^{\prime}\right)^{T} \delta \boldsymbol{r} d x=0 \\
\int_{0}^{L} \delta \boldsymbol{b}^{T}\left(\boldsymbol{\kappa}^{*}-\boldsymbol{\vartheta}^{\prime}-\boldsymbol{d}\right) d x+\int_{0}^{L} \boldsymbol{b}^{T} \mathbf{R} \delta \boldsymbol{\kappa} d x-\left[\boldsymbol{b}^{T} \delta \boldsymbol{\vartheta}\right]_{0}^{L}+\int_{0}^{L}\left(\boldsymbol{b}^{\prime}\right)^{T} \delta \boldsymbol{\vartheta} d x=0
\end{array}
$$

By adding equations (18) and (19) to (17), we obtain the modified principle of virtual work in which the variations $\delta \boldsymbol{\gamma}, \delta \boldsymbol{\kappa}, \delta \boldsymbol{\vartheta}, \delta \boldsymbol{r}, \delta \boldsymbol{a}$, and $\delta \boldsymbol{b}$ are arbitrary and independent functions of $x$. The variations $\delta \boldsymbol{r}^{0}, \delta \boldsymbol{\vartheta}^{0}, \delta \boldsymbol{r}^{L}$, and $\delta \boldsymbol{\vartheta}^{L}$ are also arbitrary and independent parameters. As the consequence of the fundamental theorem of calculus of variations [30] all the coefficients at the independent variations vanish and the following Euler-Lagrange equations of the threedimensional beam are obtained

$$
\begin{aligned}
\boldsymbol{N}-\mathbf{R}^{T} \boldsymbol{a} & =\mathbf{0} \\
\boldsymbol{M}-\mathbf{R}^{T} \boldsymbol{b} & =\mathbf{0} \\
\boldsymbol{n}+\boldsymbol{a}^{\prime} & =\mathbf{0} \\
\boldsymbol{m}+\boldsymbol{b}^{\prime}-\boldsymbol{a} \times \mathbf{R}(\gamma-\boldsymbol{c}) & =\mathbf{0} \\
\mathbf{R} \gamma-\boldsymbol{r}^{\prime}-\mathbf{R} \boldsymbol{c} & =\mathbf{0} \\
\boldsymbol{\kappa}^{*}-\boldsymbol{\vartheta}^{\prime}-\boldsymbol{d} & =\mathbf{0}
\end{aligned}
$$

along with the boundary conditions 


$$
\begin{array}{ll}
\boldsymbol{S}^{0}+\boldsymbol{a}^{0}=\mathbf{0} & \boldsymbol{S}^{L}-\boldsymbol{a}^{L}=\mathbf{0} \\
\boldsymbol{P}^{0}+\boldsymbol{b}^{0}=\mathbf{0} & \boldsymbol{P}^{L}-\boldsymbol{b}^{L}=\mathbf{0} .
\end{array}
$$

Equations (20)-(25) constitute a system of six matrix equations for six unknown vector functions $\boldsymbol{\gamma}(x), \boldsymbol{\kappa}^{*}(x), \boldsymbol{r}(x), \boldsymbol{\vartheta}(x), \boldsymbol{a}(x)$, and $\boldsymbol{b}(x)$ for a given set of loads, described by $\boldsymbol{n}(x), \boldsymbol{m}(x), \boldsymbol{S}^{0}, \boldsymbol{P}^{0}, \boldsymbol{S}^{L}$, and $\boldsymbol{P}^{L}$. Equations (22) and (23) are the force and moment equilibrium conditions. The physical meaning of the Lagrangian multipliers $\boldsymbol{a}$ and $\boldsymbol{b}$ is now obvious: $\boldsymbol{a}(x)$ is the cross-sectional force resultant at point $x ; \boldsymbol{b}(x)$ is the cross-sectional moment resultant at point $x . \boldsymbol{a}$ and $\boldsymbol{b}$ satisfy the equilibrium equations and will hence be referred to as the equilibrium force and moment. We have already introduced the crosssectional force and moment resultants as computed from the strains by the constitutive equations and these will therefore be termed the constitutive force and moment. Thus, equations (20) and (21) demand the equilibrium force and moment vectors $\boldsymbol{a}$ and $\boldsymbol{b}$ be equal to the constitutive force and moment vectors $\boldsymbol{N}$ and $\boldsymbol{M}$, respectively. These conditions yield the so-called 'consistent equilibrium at the cross-section'. Inequality of equilibrium and constitutive stresses is typical for standard finite element formulations. It may constitute a substantial source of error, especially in materially non-linear problems. An application of these important consistency conditions in the elastic-plastic finite element analysis of plane frames is presented in the paper by Vratanar and Saje [31].

Let us take that the set of equations (22)-(25) is satisfied when $\boldsymbol{n}, \boldsymbol{m}, \boldsymbol{\gamma}, \boldsymbol{\vartheta}$, and $\boldsymbol{\kappa}$ are known at any point of the centroidal line. Let us further assume that the strain vector $\boldsymbol{\gamma}$ can uniquely be determined from (15), provided that $\boldsymbol{\kappa}, \boldsymbol{\kappa}_{0}$, and $\gamma_{0}$ are known. As a result, equation (21) remains the only equation of the system (20)-(25) that still needs to be solved with the the pseudo-curvature $\boldsymbol{\kappa}^{*}(x)$ as the only unknown function. In order to connect an individual beam element to a frame structure in the physical space, the boundary conditions need to be taken into account. $\boldsymbol{a}^{L}$ and $\boldsymbol{b}^{L}$ are obtained by integration of (22) and (23), and then inserted into (26). The values of $\boldsymbol{r}^{L}$ and $\boldsymbol{\vartheta}^{L}$ are expressed by integration of $(24)-(25) \cdot \boldsymbol{r}^{L}$ and $\boldsymbol{\vartheta}^{L}$ need to be added to the boundary equations, as a consequence of the change of the variables from $\boldsymbol{r}$ and $\boldsymbol{\vartheta}$ to 
$\kappa^{*}$. The final governing equations of the three-dimensional beam read

$$
\begin{gathered}
\boldsymbol{M}_{G}(x)-\mathbf{R}^{T} \boldsymbol{b}_{g}(x)=\mathbf{0} \\
\boldsymbol{r}_{g}^{L}-\boldsymbol{r}_{g}^{0}-\int_{0}^{L} \mathbf{R}\left(\gamma_{G}-\boldsymbol{c}_{G}\right) d x=\mathbf{0} \\
\boldsymbol{\vartheta}_{g}^{L}-\boldsymbol{\vartheta}_{g}^{0}-\int_{0}^{L}\left(\boldsymbol{\kappa}_{g}^{*}-\boldsymbol{d}_{g}\right) d x=\mathbf{0} \\
\boldsymbol{S}_{g}^{0}+\boldsymbol{a}_{g}^{0}=\mathbf{0} \\
\boldsymbol{P}_{g}^{0}+\boldsymbol{b}_{g}^{0}=\mathbf{0} \\
\boldsymbol{S}_{g}^{L}-\boldsymbol{a}_{g}^{0}+\int_{0}^{L} \boldsymbol{n}_{g} d x=\mathbf{0} \\
\boldsymbol{P}_{g}^{L}-\boldsymbol{b}_{g}^{0}-\int_{0}^{L}\left[\boldsymbol{a}_{g} \times \mathbf{R}\left(\gamma_{G}-\boldsymbol{c}_{G}\right)-\boldsymbol{m}_{g}\right] d x=\mathbf{0} .
\end{gathered}
$$

\section{$5 \quad$ Numerical solution of governing equations}

\subsection{Finite element formulation}

The finite element method is used to solve equations (27)-(33). The system of non-linear governing equations of the beam thus obtained is solved by Newton's method. The discretization of equation (27) represents the crucial step. It depends on the number and the positions of the integration nodes, used for the numerical evaluation of integrals in (28)-(29) and (32)-(33). Integrals over interval $[0, L]$ are substituted by finite sums over the global integration nodes $x_{p}$

$$
\int_{0}^{L} \boldsymbol{f}(x) d x \rightarrow \sum_{p=1}^{N} w_{p} \boldsymbol{f}^{p} .
$$

Here $w_{p}$ are the weights of the quadrature method used, and $\boldsymbol{f}^{p}$ the values $\boldsymbol{f}\left(x_{p}\right)$ of integrand at the integration nodes. We have chosen to make equation (27) be satisfied solely at integration nodes $x_{p}$ :

$$
\boldsymbol{M}_{G}\left(x_{p}\right)-\mathbf{R}^{T} \boldsymbol{b}_{g}\left(x_{p}\right)=\mathbf{0} .
$$

Thus, the finite element formulation used here is a collocation method.

Remark 4 Observe that the same result follows from the Galerkin-type of the finite element method as a special case if the interpolation is chosen through the integration nodes.

The resulting discretized equations constitute a system of $N+6$ non-linear vector equations of a beam element, where $N$ denotes the number of inte- 
gration nodes used. The vector unknowns are $\boldsymbol{r}^{0}, \boldsymbol{\vartheta}^{0}, \boldsymbol{a}^{0}, \boldsymbol{b}^{0}, \boldsymbol{r}^{L}, \boldsymbol{\vartheta}^{L}, \boldsymbol{\kappa}^{* p}$ $(p=1,2, \ldots, N)$.

In order to determine the values of the dependent quantities $\boldsymbol{a}(x), \boldsymbol{b}(x)$, $\boldsymbol{r}(x)$, and $\boldsymbol{\vartheta}(x)$ at the nodes $x_{p}$ from the nodal pseudo-curvatures $\boldsymbol{\kappa}^{* p}(p=$ $1,2, \ldots, N)$, a set of additional local (or internal) integrals needs to be evaluated numerically. A low order local integration, which uses only global integration nodes, could be used. However, we do not wish to restrict the order of the local numerical integration. For that purpose, some interpolation of the pseudo-curvature must be introduced. At this point we need to emphasize that the configuration space of the unknown $\boldsymbol{\kappa}^{*}$ is non-linear because of the multiplicative (non-additive) nature of rotations and the related quantities. On the other hand, the space of the variations $\delta \boldsymbol{\kappa}^{*}$ is linear, so the interpolation of the variations $\delta \boldsymbol{\kappa}^{*}$ in a classical form of the linear combination is theoretically admissible. Therefore, the interpolation of $\delta \boldsymbol{\kappa}^{*}$ is used in the form

$$
\delta \boldsymbol{\kappa}^{*}(x)=\sum_{p=1}^{N} I_{p}(x) \delta \boldsymbol{\kappa}^{* p} .
$$

$I_{p}(x)(p=1,2, \ldots, N)$ are the interpolation functions (not necessarily polynomials) through the integration nodes $x_{p}$.

Remark 5 When using the interpolation of $\delta \boldsymbol{\kappa}^{*}$ and not that of $\boldsymbol{\kappa}^{*}$, we should recast equation (29) into its variational (weak) form. Nevertheless, the correct boundary values are obtained, since any increment $\delta \boldsymbol{\kappa}^{*}$ preserves kinematically exact boundary incremental rotations.

The interpolation of $\delta \boldsymbol{\kappa}^{*}(x)$ allows us to introduce the numerical integration of any order over closed intervals $\left[0, x_{p}\right]$. In the computer implementation of the algorithm, an effective step-by-step computation of the local integrals was used. In each integration step, solely the quadrature between the two subsequent global integration nodes is applied and the result is then added to the previously obtained one. An example showing an element using the 4-node global Gaussian integration $(N=4)$ and the 3-point local Gaussian integration between the global integration nodes is illustrated in Figure 2.

- interpolation, collocation, global integration nodes

- local integration points

I boundary points

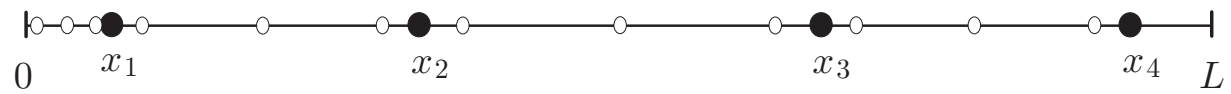

Fig. 2. Interpolation, collocation and global integration nodes, and local integration points. 


\subsection{Newton's iteration method}

The non-linearity of the configuration space of the beam requires a special care in applying Newton's method to the problem. The linearization is made in the sense of the first variation of functionals. This way the variations of the unknowns become equal to the iterative increments of the unknowns. The variations of the unknowns are elements of the tangent space. Thus an update procedure needs to be applied to map the unknowns from the tangent space onto the configuration space.

Varying the discrete system of non-linear equations (28)-(34) assembled for all elements of a system, gives the Jacobian matrix of the system (or the tangent stiffness matrix). Following Newton's iteration scheme, a system of linear equations is solved at each iteration step $n=0,1,2, \ldots$

$$
\mathbf{K}^{[n]} \delta \boldsymbol{y}=-\boldsymbol{h}^{[n]},
$$

where $\mathbf{K}^{[n]}$ is the global tangent stiffness matrix, $\boldsymbol{h}^{[n]}$ is the residual vector of equations (28)-(34), both in iteration $n$, and $\delta \boldsymbol{y}$ is a vector of corrections, which, in classical Newton's method in linear vector spaces, is added to the previous solution iterate vector $\boldsymbol{y}^{[n]}$. The non-linearity of the configuration space, on the other hand, requires a non-classical update procedure. Its basic idea is illustrated in Figure 3.

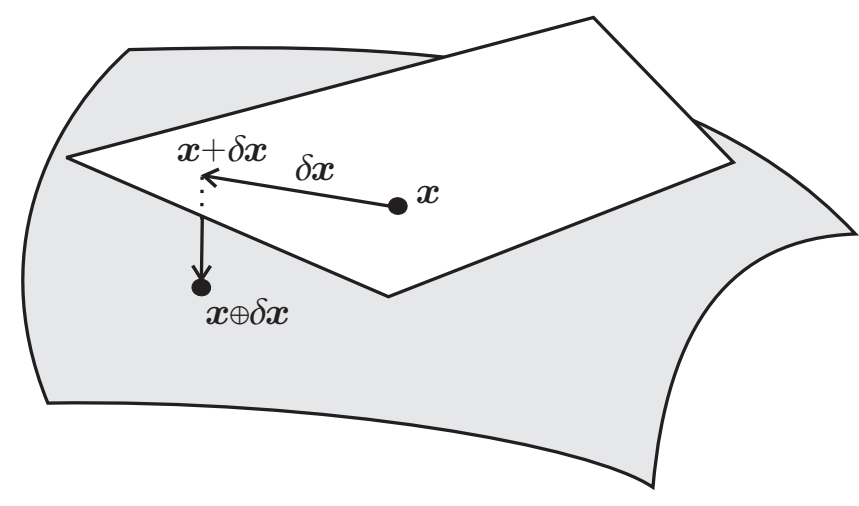

Fig. 3. Update procedure in a non-linear configuration space.

The non-linear configuration space is symbolically illustrated by a curved surface. The iterative approximation to the solution vector, $\boldsymbol{x}$, is an element of the non-linear space. The variation of the solution vector, $\delta \boldsymbol{x}$, is an element of a linear space, here symbolically illustrated by a tangent plane. A new approximation of the solution is obtained, first by determining a new correction vector, point $\boldsymbol{x}+\delta \boldsymbol{x}$ on the tangent plane, and then by projecting the point onto the configuration space. 
As a result of an iteration step, the corrections of the unknowns are obtained, $\delta \boldsymbol{r}^{0}, \delta \boldsymbol{\vartheta}^{0}, \delta \boldsymbol{a}^{0}, \delta \boldsymbol{b}^{0}, \delta \boldsymbol{r}^{L}, \delta \boldsymbol{\vartheta}^{L}, \delta \boldsymbol{\kappa}^{* p}$. The position vector and stress resultants are linear quantities. Therefore, new values are obtained by a simple addition of the corrections to the previous values:

$$
\begin{array}{lll}
\boldsymbol{r}^{0[n+1]}=\boldsymbol{r}^{0[n]}+\delta \boldsymbol{r}^{0} & \boldsymbol{r}^{L[n+1]}=\boldsymbol{r}^{L[n]}+\delta \boldsymbol{r}^{L} \\
\boldsymbol{a}^{0[n+1]}=\boldsymbol{a}^{0[n]}+\delta \boldsymbol{a}^{0} & \boldsymbol{b}^{0[n+1]}=\boldsymbol{b}^{0[n]}+\delta \boldsymbol{b}^{0} .
\end{array}
$$

The remaining quantities are not additive. The update of boundary rotational vectors is as follows: first, corrections of boundary rotation matrices, $\delta \mathbf{R}^{0}, \delta \mathbf{R}^{L}$ are calculated from $\delta \boldsymbol{\vartheta}^{0}$ and $\delta \boldsymbol{\vartheta}^{L}$ with the Rodrigues formula (4). Then new boundary rotation matrices are obtained by matrix multiplication:

$$
\begin{aligned}
& \mathbf{R}^{0[n+1]}=\delta \mathbf{R}^{0} \mathbf{R}^{0[n]} \\
& \mathbf{R}^{L[n+1]}=\delta \mathbf{R}^{L} \mathbf{R}^{L[n]} .
\end{aligned}
$$

Finally, the Spurrier algorithm [27] is used to extract new boundary rotational vectors $\boldsymbol{\vartheta}^{0[n+1]}$ and $\boldsymbol{\vartheta}^{L[n+1]}$ from $\mathbf{R}^{0[n+1]}$ and $\mathbf{R}^{L[n+1]}$. The algorithm first obtains the rotational quaternion from the rotation matrix. From the rotational quaternion, $\arccos \vartheta$ is then evaluated and angle $\vartheta$ in the interval $[0,2 \pi)$ uniquely obtained. $\vartheta$ from this interval fully suffices to describe boundary rotations of any magnitude in the tangent stiffness matrix because the rotational vector is invariant with respect to an additional rotation of magnitude $2 n \pi$.

The crucial part of our procedure is the update of the rotation and the pseudocurvature vectors at points along the beam axis. In contrast to the update procedure for the boundary rotations, the update of the rotations at internal points does not require the extraction of the rotational vector from the rotation matrix. The corrections $\delta \boldsymbol{\kappa}^{*}$ along the axis of the beam are first obtained by the interpolation (35)

$$
\delta \boldsymbol{\kappa}^{*}(x)=\sum_{p=1}^{N} I_{p}(x) \delta \boldsymbol{\kappa}^{* p} .
$$

The application of (37) in the weak form of (29) yields the corrections of the rotational vector

$$
\delta \boldsymbol{\vartheta}(x)=\delta \boldsymbol{\vartheta}^{0}+\sum_{p=1}^{N} \delta \boldsymbol{\kappa}^{* p} \int_{0}^{x} I_{p}(\xi) d \xi
$$

From (38) the correction of the rotation matrix, $\delta \mathbf{R}(x)$, is obtained by the Rodrigues formula (4). New values of the rotation matrix are calculated by matrix multiplication

$$
\mathbf{R}^{[n+1]}(x)=\delta \mathbf{R}(x) \mathbf{R}^{[n]}(x) .
$$

Observe that the rotations of any magnitude including those of magnitude $\vartheta=2 n \pi$, can be dealt with this algorithm in a stable manner. 
The update of the deformation quantity $\boldsymbol{\kappa}(x)$ is based on the additivity of the pseudo-curvature vector $\boldsymbol{\kappa}$, when expressed with respect to the material basis (see, e.g., Shabana [22]). The correction of the vector $\boldsymbol{\kappa}$ in the material basis is obtained by the application of the transformation matrix (12) on the correction of $\boldsymbol{\kappa}^{*}$ (see equation (13)) and by the coordinate transformation. The formula for the update of $\boldsymbol{\kappa}(x)$ reads

$$
\boldsymbol{\kappa}^{[n+1]}(x)=\boldsymbol{\kappa}^{[n]}(x)+\mathbf{R}^{[n+1] T}(x) \mathbf{T}(\delta \boldsymbol{\vartheta}(x)) \delta \boldsymbol{\kappa}^{*}
$$

\section{$6 \quad$ Numerical examples}

In this section, we present several numerical examples in order to demonstrate the performance and accuracy of the proposed formulation. To enable the comparison with other formulations, a linear elastic material is employed in all numerical examples. The operators $\mathcal{C}_{N}$ and $\mathcal{C}_{M}$ in (15)-(16) are taken to be diagonal matrices

$$
\mathcal{C}_{N}=\left[\begin{array}{ccc}
E A_{1} & 0 & 0 \\
0 & G A_{2} & 0 \\
0 & 0 & G A_{3}
\end{array}\right] \quad \mathcal{C}_{M}=\left[\begin{array}{ccc}
G J_{1} & 0 & 0 \\
0 & E J_{2} & 0 \\
0 & 0 & E J_{3}
\end{array}\right] .
$$

Here $E$ and $G$ denote elastic and shear moduli of material; $A_{1}$ is the crosssectional area, $J_{1}$ is the torsional inertial moment of the cross-section; $A_{2}$ and $A_{3}$ are the shear areas in the principal inertial directions 2 and 3 of the crosssection; $J_{2}$ and $J_{3}$ are the cross-sectional inertial moments about its principal directions 2 and 3.

Finite elements with different degrees of interpolation polynomials are used in order to investigate the influence of the number of interpolation (and global integration) nodes and the order of the local integration on the accuracy of numerical solutions. A particular type of the element is marked by the symbol ' $E$ ' and equipped with two integer values, $E_{N-M} ; N$ is the number of interpolation nodes, and $M$ is the number of additional internal points between the two subsequent global integration nodes used for the local integration. Recall that the incremental pseudo-curvatures are interpolated by the polynomials of degree $N-1$. Each element has thus $3 \cdot(6+N)$ degrees of freedom.

The quadratic convergence of Newton's method was achieved in all numerical examples. The iteration was terminated when the Euclidean norm of the vector of nodal unknowns, $\|\delta \boldsymbol{y}\|_{2}$, and of the vector of unbalanced residual forces, $\|\boldsymbol{h}\|_{2}$, was less than $10^{-11}$. 


\subsection{Lateral buckling of a cantilever}

We consider a straight, inextensible, shear and in-plane-bending stiff cantilever, subjected to the point force at its free end (see Figure 4). The lateral out-of-plane buckling load $F_{c}$ is sought. The numerical results are compared with the analytical solution, provided by Timoshenko and Gere [29].

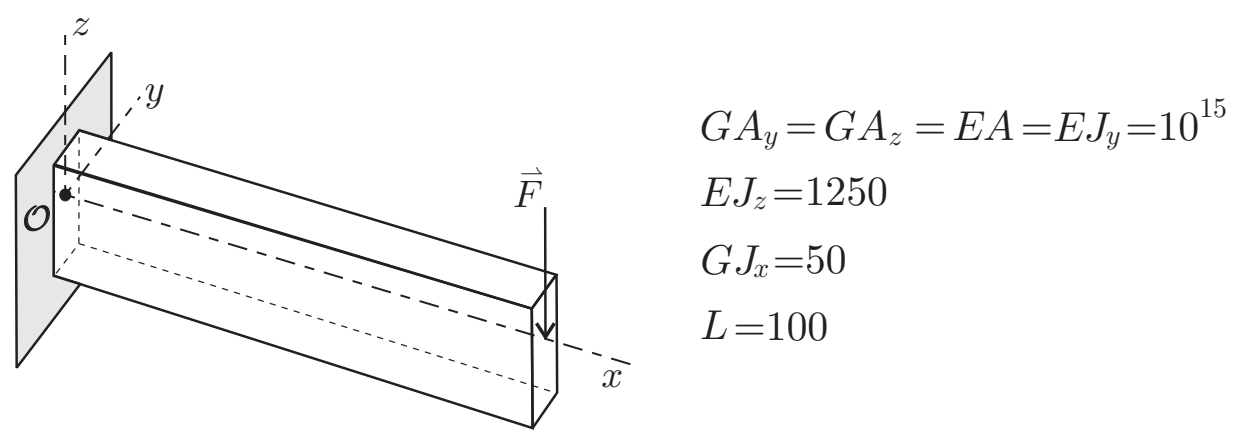

Fig. 4. Lateral buckling of a cantilever.

We obtain the buckling load iteratively by employing the condition that the critical load represents the load at which the tangent stiffness matrix of the cantilever becomes singular. The inextensibility, and shear and in-plane stiffnesses are approximated by large values $10^{15}$ for $G A_{y}, G A_{z}, E A$, and $E J_{y}$ (Figure 4).

In Table 1 the influence of the number of elements, the number of interpolation nodes per element, and the number of local integration points on the critical load is displayed. We can see that the increasing of the number of interpolation nodes gives a higher rate of convergence and better accuracy than the increase of the number of elements. The influence of the order of the local integration is also displayed, although its effect here is only minor.

When a single element with two nodes is employed, a $12 \%$ error is found. Increasing the number of two-node elements yields substantially more accurate results. To obtain a nine-digit accurate solution, 20 elements with 3 nodes are required; such a mesh has 426 degrees of freedom. Equally accurate results are obtained if one 8-node element with 42 degrees of freedom is used. We should point out the similarity of our results and those obtained by Jelenić and Saje [13]. The results fully agree, the only difference being that the formulation by Jelenić and Saje requires a one degree higher interpolation polynomial.

It is thus obvious that a few sufficiently high-order elements are more efficient than many low-order elements, provided that the high order elements do not exhibit the locking, which is the case here. Note that the reduced integration often used in practice, is not a preferable solution for high order elements, because it may reduce the accuracy of the element by a large extent. 
Table 1

The out-of-plane buckling load.

\begin{tabular}{llllll}
\hline t.e. & n.d.o.f. & $n_{e}=1$ & $n_{e}=2$ & $n_{e}=5$ & $n_{e}=20$ \\
\hline$E_{2-2}$ & & 0.112219000 & 0.101432352 & 0.100349434 & 0.100315118 \\
$E_{2-10}$ & & 0.112219000 & 0.101432352 & 0.100349434 & 0.100315118 \\
\hline$E_{3-2}$ & 426 & 0.101375990 & 0.100349169 & 0.100315163 & 0.100314984 \\
$E_{3-3}$ & 426 & 0.101375990 & 0.100349169 & 0.100315163 & 0.100314984 \\
$E_{3-10}$ & 426 & 0.101375990 & 0.100349169 & 0.100315163 & 0.100314984 \\
\hline$E_{6-2}$ & 156 & 0.100315089 & 0.100314970 & 0.100314980 & \\
$E_{6-6}$ & 156 & 0.100315404 & 0.100314984 & 0.100314984 & \\
$E_{6-10}$ & 156 & 0.100315404 & 0.100314984 & 0.100314984 & \\
\hline$E_{7-2}$ & 72 & 0.100314839 & 0.100314981 & & \\
$E_{7-7}$ & 72 & 0.100315000 & 0.100314984 & & \\
$E_{7-10}$ & 72 & 0.100315000 & 0.100314984 & & \\
\hline$E_{8-2}$ & 42 & 0.100314980 & & & \\
$E_{8-5}$ & 42 & 0.100314983 & & & \\
$E_{8-8}$ & 42 & 0.100314983 & & & \\
\hline analytical solution & $\mathbf{0 . 1 0 0 3 1 4 9 8 4}$ & & \\
\hline
\end{tabular}

t.e. $=$ type of element, n.d.o.f. $=$ number of degrees of freedom, $n_{e}=$ number of elements

\subsection{The in-plane stability of a deep circular arch}

We study the in-plane buckling-type of stability of an elastic beam with the centroidal axis shaped in the form of the 215 degree circular arc with radius $R=100$. This example is characterized by large pre-buckling displacements and rotations. The problem has been widely studied. As the reference solution, the result of DaDeppo and Schmidt [7] is usually stated. In contrast to many authors who used the finite element method, the finite difference method was used in [7]. As estimated in [7], the error of their solution is roughly $0.03 \%$, thus the results given in [7] are correct to three digits $\left(F_{\mathrm{cr}}=897\right)$.

In our study we have chosen the global coordinate system, in which the centroidal axis lies in the plane $x z$ and the reference point $(0,0,0)$ coincides with the center of the circle. Concentrated force $\vec{F}=-F \vec{e}_{z}$ acts at the point $(0,0, R)$. The values of the remaining material and geometrical parameters are $E J_{y}=E J_{z}=G J_{t}=10^{6}, E A=G A_{x}=G A_{y}=10^{8}$. The comparisons between the results of the present formulation and the results of various other 

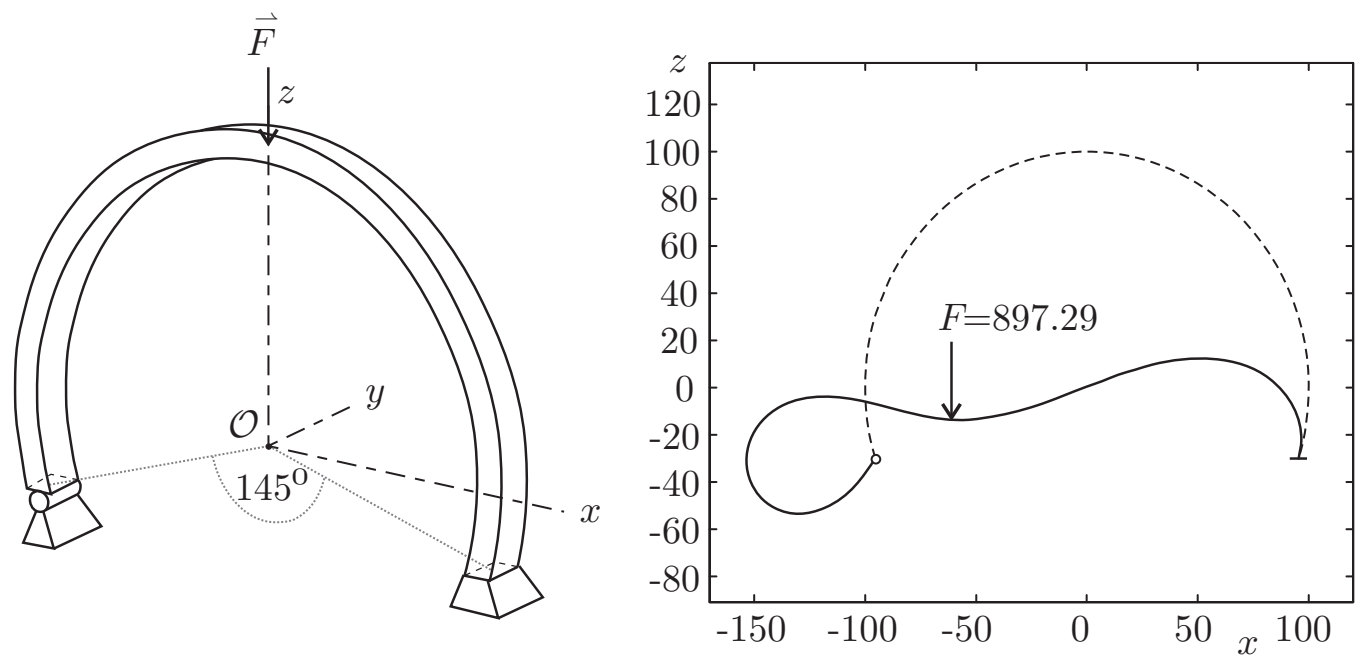

Fig. 5. Deep circular arch and deformed axis of centroids at the critical point.

formulations are presented in Table 2.

Table 2

The comparison between different results of the critical force of a deep circular arch.

\begin{tabular}{rrrrrrrrrr}
$n_{e}$ & $E_{2-2}$ & $E_{3-2}$ & $E_{8-3}$ & $E_{2-2}$ & $E_{3-2}$ & {$[21]$} & {$[9]$} & {$[9]$} & {$[24]$} \\
& curved & curved & curved & str. & str. & curved & curved & str. & str. \\
\hline 2 & & & 896.57 & & & 904.53 & & & \\
4 & & & 897.29 & & & 897.29 & & & \\
6 & 903.22 & 897.74 & 897.29 & & & & & & \\
12 & 897.71 & 897.30 & & & & & & & \\
20 & & & & 907.31 & 907.30 & & 897.5 & 906 & \\
24 & 897.32 & 897.29 & & & & & & & \\
40 & & & & 899.80 & 899.80 & & & & 905.28 \\
48 & 897.29 & 897.29 & & & & & & & \\
\hline
\end{tabular}

$E=$ type of element, $n_{e}=$ number of elements, str.=initially straight element

The present results and the results from [21] are found to be the most accurate, while employing the least number of degrees of freedom. The efficiency of these two formulations is also the result of the fact that initial curvature of the elements is considered. This is confirmed in the fourth and fifth column of results of Table 2, where you can see the results of the polygonal approximation of the arc for initially straight elements $E_{2-2}$ and $E_{3-2}$. The present results are obtained by the use of several types of elements, while other studies use 9-node [21], 3-node [9] and linear finite elements [24]. In Table 2 we can see how accurate the present formulation is, not only for high order elements but also for low-order curved elements $E_{2-2}$ and $E_{3-2}$. Note that the high-order 
element $E_{8-3}$ compared to lower order elements, needs less degrees of freedom for the solution of equal accuracy.

\subsection{Free-end displacements of pretwisted cantilevers}

In this numerical example we consider the influence of a pretwist on a free-end displacement of a cantilever. The beams are clamped at one end and loaded by the force at the other. The centroidal axis is straight at the beginning, but the cross sections are twisted about the centroidal axis. The initial rotation along the length of the beam is therefore described by the rotation angle as a function of arc-length $x$. The cantilever with linear dependence between the arc-length and the rotation angle, and with the $\frac{1}{2} \pi$ free-end rotation was analyzed by Tabarrok et al. [28]. The material and geometrical properties of the beam are shown in Figure 6.

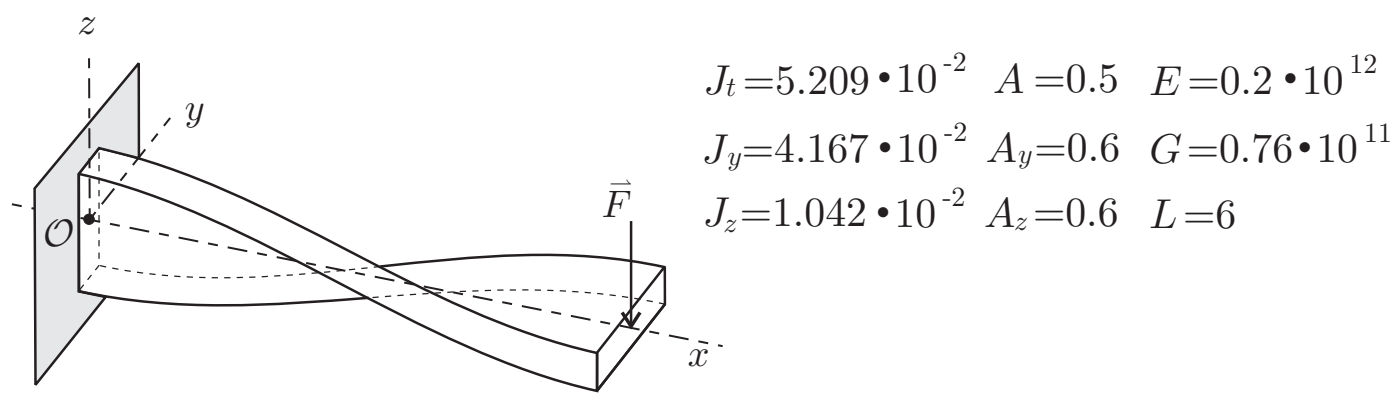

Fig. 6. Pretwisted beam for an angle of $\pi / 2$.

In Table 3 the displacements $u_{x}, u_{y}$, and $u_{z}$ of the free-end of the cantilever under the free-end force $F=-3000$ are given. The results of the present formulation are compared to those given in [28]. Only one 5-node element $E_{5-2}$ suffices to obtain the displacements which are accurate to 5 significant figures. This compares favourably with the result in [28], where 20 elements were used. The same accuracy is obtained when using the mesh of 5 linear $\left(E_{2-2}\right)$ elements; in this case however, about three times as many degrees of freedom are needed. The comparison between different types of elements used reveals the rapid convergence of the present formulation.

It is interesting to display the end displacement as a function of the direction of the applied force. The results are shown as polar graphs in Figure 7, where the projection of the resulting end-displacement onto the direction of the applied force as a function of the force direction is drawn.

Several cases are considered in Figure 7. In all these cases, the cantilever is initially twisted and subjected to point load at its free end. The load 3000 is applied while its direction, $(0, \sin \varphi, \cos \varphi) ; \varphi \in[0,2 \pi]$, alters (rotates) in the $y z$ plane. For an angle $\varphi$, which defines the direction of the applied force, the 
Table 3

Free-end displacements of an $\pi / 2$-pretwisted cantilever.

\begin{tabular}{rrrrrr}
\hline$n_{e}$ & t.e. & n.d.o.f. & $-u_{z} \cdot 10^{-5}$ & $-u_{x} \cdot 10^{-10}$ & $-u_{y} \cdot 10^{-5}$ \\
\hline 1 & $E_{2-2}$ & 24 & 3.867550 & 2.149780 & 2.395749 \\
1 & $E_{3-2}$ & 27 & 4.165452 & 2.463750 & 2.194676 \\
1 & $E_{5-2}$ & 33 & 4.155854 & 2.451590 & 2.208035 \\
1 & $E_{8-3}$ & 42 & 4.155853 & 2.451580 & 2.208039 \\
\hline 3 & $E_{2-2}$ & 60 & 4.153760 & 2.449610 & 2.208380 \\
3 & $E_{3-2}$ & 69 & 4.155861 & 2.451610 & 2.208031 \\
3 & $E_{5-2}$ & 87 & 4.155853 & 2.451610 & 2.208039 \\
\hline 5 & $E_{2-2}$ & 96 & 4.155592 & 2.451350 & 2.208073 \\
5 & $E_{3-2}$ & 111 & 4.155853 & 2.451630 & 2.208039 \\
5 & $E_{5-2}$ & 141 & 4.155853 & 2.451610 & 2.208039 \\
\hline 10 & $E_{2-2}$ & 186 & 4.155837 & 2.451600 & 2.208041 \\
10 & $E_{3-2}$ & 216 & 4.155853 & 2.451620 & 2.208039 \\
\hline
\end{tabular}

Tabarrok et al. [28]

$\begin{array}{ll}12 & 4.146810 \\ 20 & 4.157768\end{array}$

$n_{e}=$ number of elements, t.e.=type of element,

n.d.o.f. $=$ number of degrees of freedom

corresponding value of projection of the displacement is drawn. The angledisplacement diagram is obviously not symmetric about the axes $\varphi=0(\varphi=$ $\pi)$ and $\varphi=\frac{\pi}{2}\left(\varphi=\frac{3 \pi}{2}\right)$. This asymmetry is the consequence of the initial asymmetric geometric shape. The polar graphs clearly show the influence of the total free-end twist on the bending stiffness of the pretwisted beam. The beam with $\psi=\frac{\pi}{2}$ is, compared to the straight (non-twisted) beam, relatively stiffer in the $y$-direction and more flexible in the $z$-direction. Besides that, the largest end displacement of the $\frac{\pi}{2}$-twisted beam occurs not when the force is applied in $y$ (out-of-plane) direction, but when it is applied at the angle of roughtly 25 degrees. The more we increase the free-end twist angle, the stiffer the beam becomes in the $y$-direction. In the case of $10 \pi$ twist, the angledisplacement graph becomes almost a circle.

We should be aware of the difference between the direction of the applied force and the direction of the resulting displacement in the $y z$ plane. In polar graphs in Figure 7, the projection of the displacement onto the force direction was 


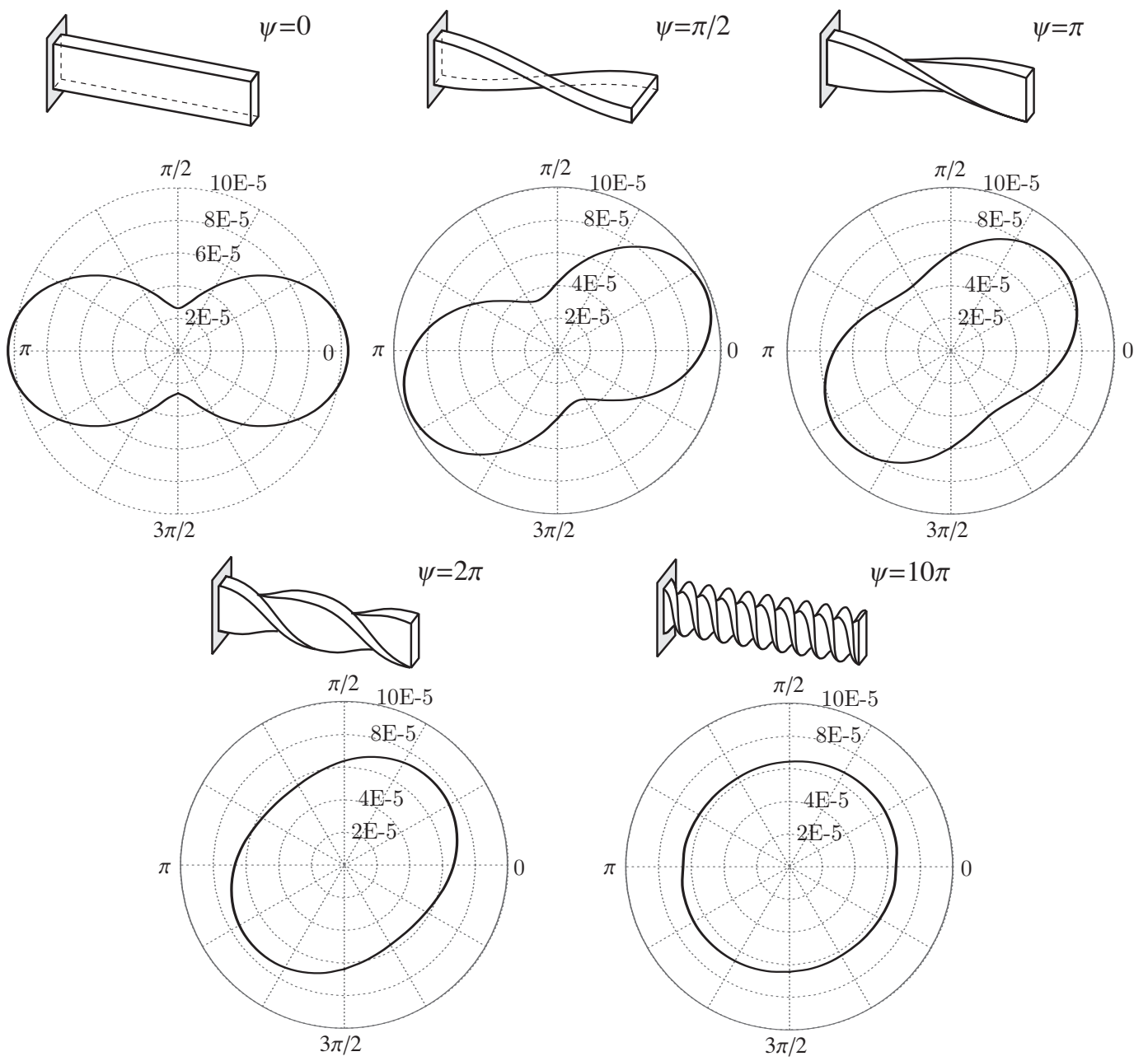

Fig. 7. Free-end displacements in the direction of the applied force for several pretwisted beams.

drawn. In general the resulting displacement in the $y z$ plane is not parallel to the direction of the applied force. In Figure 8 the difference of directions is illustrated for several initially twisted beams. For an angle of the applied force $\varphi$, the absolute difference between $\varphi$ and the angle of the resulting displacement vector is drawn (in radian). For the initially straight beam, the difference of angles vanishes only for the forces acting in the directions of the axes $y$ and $z$. For other directions of the applied force, the direction of the resulting displacement is rather different. Its value for $\varphi=\frac{\pi}{6}$ is approximately 36 degrees. If the pretwisting of the beam is increased, the diagram of the absolute difference between both angles rotates left-wise and becomes smaller, but the general shape of the curve remains the same.

Another important detail needs to be discussed. In all of the previous examples, the angle of pretwist was a linear function of the arc-length of the line of centroids. Let us now consider a beam with the non-linear dependence. Let us take the $\frac{\pi}{2}$-pretwisted beam with straight edges (Figure 9), probably the 

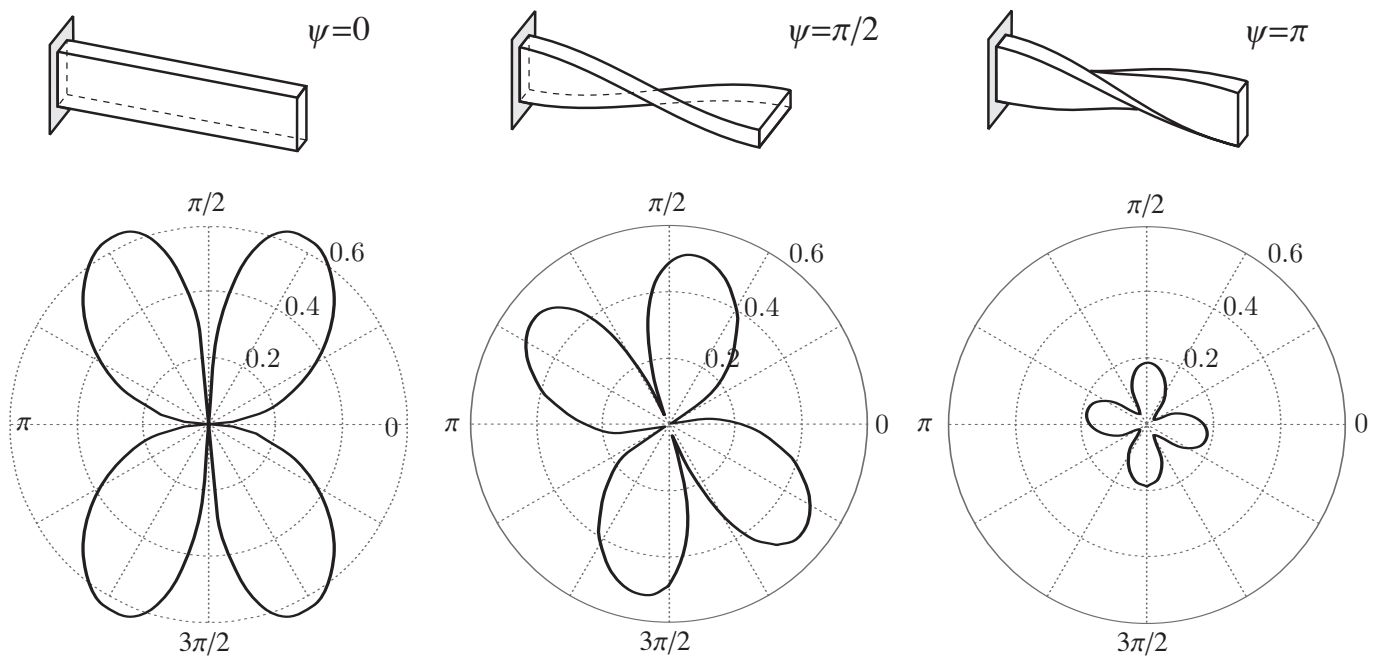

Fig. 8. The absolute difference between the direction angle of the applied force and the angle of the resulting displacement vector for several pretwisted beams, in radian.
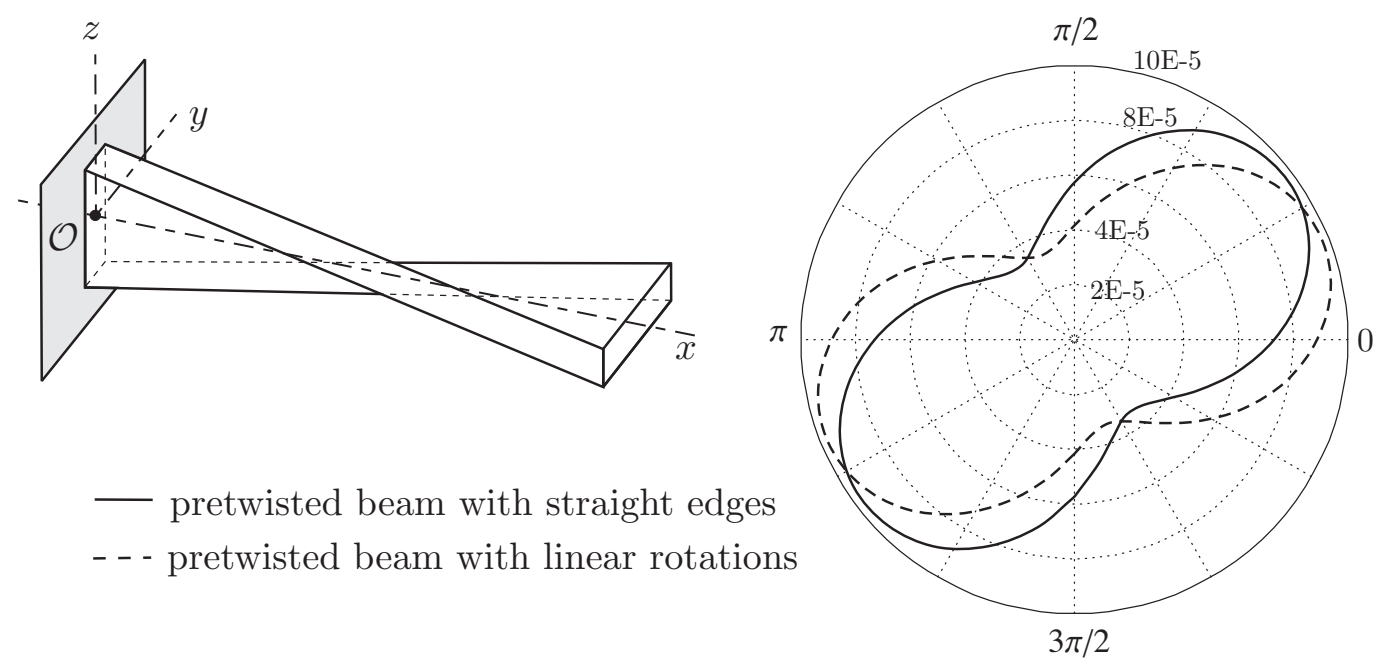

Fig. 9. The $\pi / 2$-pretwisted beam with straight edges (non-linear pretwist along length of the beam).

simplest example of a non-linearly pretwisted beam. The same load cases are considered as previously and the corresponding polar graph is evaluated. On the right-hand side of Figure 9, the graph is compared to the one obtained by the linearly pretwisted beam. Notice that, in principle, similar behaviour is found yet with different values. 


\section{Conclusions}

We presented a new finite element formulation of the geometrically exact threedimensional beam theory based on the interpolation of pseudo-curvature. The formulation accounts for large displacements, rotations and large strains without any practical limitations. These are the essential points of the formulation:

(i) A modified principle of virtual work is proposed, in which the only unknown function is the variation of the pseudo-curvature vector. Thus the only function that needs to be interpolated is the iterative increment (or the variation) of the pseudo-curvature vector $\delta \boldsymbol{\kappa}^{*}$; this vector represents the energy complement to the moment vector $\boldsymbol{M}$ given with respect to the spatial basis.

(ii) Displacements and rotational vectors (or their variations) are not interpolated.

(iii) The consistency condition that the equilibrium and the constitutive internal force and moment vectors are equal, is satisfied at the interpolation points. This considerably improves the accuracy of the internal forces and moments in materially non-linear problems.

(iv) The determination of internal forces and moments does not require the differentiation. Therefore the accuracy of the internal forces and moments is of the same order as the accuracy of the basic variable - the pseudocurvature. This is an important advantage compared to formulations where the derivatives are needed for the evaluation of internal forces.

(v) The matrices of a finite element are derived directly with respect to the global coordinate system. The coordinate transformation from the local to the global system is thus avoided. An arbitrary curvature and extensional deformation of the beam axis, and non-orthogonality of cross-sections with respect to the axis can be prescribed at the initial unloaded configuration.

(vi) The present finite elements are free of locking.

(vii) A number of finite elements of different order have been tested by various numerical examples. A rapid convergence is a characteristic of all elements.

\section{Acknowledgment}

This work was supported by the Ministry of Education, Science and Sport of the Republic of Slovenia through the grant S2-792-016/19121. The support is gratefully acknowledged. 


\section{References}

[1] J. Argyris, "An excursion into large rotations", Comput. Methods Appl. Mech. Eng. 32, 85-155, 1982.

[2] S.N. Atluri, A. Cazzani, "Rotations in computational solid mechanics", Arch. Comput. Methods in Eng. 2, 49-138, 1995.

[3] A. Cardona, M. Géradin, "A beam finite element non-linear theory with finite rotations", Int. J. Numer. Methods Eng. 26, 2403-2438, 1988.

[4] M.A. Crisfield, "A consistent co-rotational formulation for non-linear, threedimensional beam elements", Comp. Meth. Appl. Mech. Engng. 81, 131-150, 1990.

[5] M.A. Crisfield, "Non-linear Finite Element Analysis of Solids and Structures. Volume 2: Advanced Topics", Wiley \& Sons, Chichester, 1997.

[6] M.A. Crisfield, G. Jelenić, "Objectivity of strain measures in the geometrically exact three-dimensional beam theory and its finite-element implementation", Proc. Roy. Soc. London A 455, 1125-1147, 1999.

[7] D.A. DaDeppo, R. Schmidt, "Instability of clamped-hinged circular arches subjected to point load", ASME J. Appl. Mech. 97, 894-896, 1975.

[8] M. Géradin, D. Rixen, "Parametrization of finite rotations in computational dynamics: a review", Revue européenne des éléments finis 4, 497-553, 1995.

[9] A. Ibrahimbegovic, "On the finite element implementation of geometrically nonlinear Reissner's beam theory: 3d curved beam element", Comput. Methods Appl. Mech. Eng. 122, 11-26, 1995.

[10] A. Ibrahimbegovic, "On the choice of finite rotation parameters", Comput. Methods Appl. Mech. Eng. 149, 49-71, 1997.

[11] M. Iura, S.N. Atluri, "Dynamic analysis of finitely stretched and rotated threedimensional space-curved beams", Comput. Struct. 29, 875-889, 1988.

[12] M. Iura, S.N. Atluri, "On a consistent theory and variational formulation of finitely stretched and rotated 3-D space-curved beams", Comput. Mech. 4, 7388, 1989.

[13] G. Jelenić, M. Saje, "A kinematically exact space finite strain beam model-finite element formulation by generalized virtual work principle", Comput. Methods Appl. Mech. Eng. 120, 131-161, 1995.

[14] G. Jelenić, M.A. Crisfield, "Geometrically exact 3D beam theory: implementation of a strain-invariant finite element for statics and dynamics", Comput. Methods Appl. Mech. Eng. 171, 141-171, 1999.

[15] M. Li, "The finite deformation theory for beam, plate and shell. Part III. The three-dimensional beam theory and the FE formulation", Comput. Methods Appl. Mech. Eng. 162, 287-300, 1998.

[16] F.A. Mcrobie, J. Lasenby, "Simo-Vu Quoc rods using Clifford Algebra", Int. J. Numer. Methods Eng. 45, 337-398, 1999.

[17] B. Nour-Omid, C.C. Rankin, "Finite rotation analysis and consistent linearization using projectors", Comput. Methods Appl. Mech. Eng. 93, 353384, 1991.

[18] I. Planinc, M. Saje, B. Čas, "On the local stability condition in the planar beam finite element", Structural Engineering and Mechanics 12, 507-526, 2001. 
[19] I. Planinc, S. Bratina, F. Saje, M. Saje, "A kinematically exact FE formulation of planar reinforced concrete frames based on pseudocurvature", ZAMM (J. Appl. Math. Mech.), 81, 881-882, 2001.

[20] E. Reissner, "On finite deformation of space-curved beams", J. Appl. Math. Phys. 32, 734-744, 1981.

[21] M. Saje, G. Turk, A. Kalagasidu, B. Vratanar, "A kinematically exact finite element formulation of elastic-plastic curved beams", Comput. Struct. 67, 197214, 1998.

[22] A.A. Shabana, "Dynamics of Multibody Systems", Second Edition. Cambridge University Press, 1998.

[23] J.C. Simo, "A finite strain beam formulation. The three-dimensional dynamic problem. Part I", Comput. Methods Appl. Mech. Eng. 49, 55-70, 1985.

[24] J.C. Simo, L. Vu-Quoc, "A three-dimensional finite-strain rod model. Part II: Computational aspects", Comput. Methods Appl. Mech. Eng. 58, 79-116, 1986.

[25] W.M. Smoleński, "Statically and kinematically exact nonlinear theory of rods and its numerical verification", Comput. Methods Appl. Mech. Eng. 178, 89113, 1999.

[26] I.S. Sokolnikoff, "Tensor analysis", John Wiley \& Sons, New York, 1951.

[27] R.A. Spurrier, "Comment on "Singularity-free extraction of a quaternion from a direction-cosine matrix"”, J. Spacecraft 15, 255, 1978.

[28] B. Tabarrok, M. Farshad, H. Yi, "Finite element formulation of spatially curved and twisted rods", Comput. Methods Appl. Mech. Eng. 70, 275-299, 1988.

[29] S.P. Timoshenko, J.M. Gere, "Theory of Elastic Stability", McGraw-Hill, New York, 1961.

[30] J.L. Troutman, "Variational Calculus with Elementary Convexity", SpringerVerlag, New York, 1983.

[31] B. Vratanar, M. Saje, "A consistent equilibrium in a cross-section of an elasticplastic beam", Int. J. Solids Structures 36, 311-337, 1999.

[32] J.P. Ward, "Quaternions and Cayley Numbers", Kluwer Academic Publishers, Dordrecht, 1997.

[33] K. Washizu, "Variational Methods in Elasticity and Plasticity", Pergamon Press, Oxford, 1975. 